\title{
Superprism effect in low-contrast photonic crystals
}

\author{
Adam Filipkowski, ${ }^{2}$ Ryszard Buczyński, ${ }^{* 1,2}$, Ireneusz Kujawa, ${ }^{2}$ Dariusz Pysz, ${ }^{2}$ Andrew Waddie, ${ }^{3}$ \\ Mohammad R. Taghizadeh, ${ }^{3}$ and Ryszard Stępień ${ }^{2}$ \\ ${ }^{1}$ Faculty of Physics, University of Warsaw, Pasteura 7, 02-093 Warsaw, Poland, \\ ${ }^{2}$ Institute of Electronic Materials Technology, Wólczyńska 133, 01-919 Warsaw, Poland \\ ${ }^{3}$ School of Engineering and Physical Sciences, Heriot-Watt University, Edinburgh EH14 4AS, Scotland, UK
}

Received March 18, 2010; accepted March 29, 2010; published March 31, 2010

\begin{abstract}
In this paper we study a superprism effect in a low contrast volumetric photonic crystal. The photonic crystals considered in the simulations have hexagonal lattices and a refractive index difference of 0.1 for double glass crystals and 0.6 for air-glass structures. The simulations show the existence of a superprism effect in both types of structures for an incident wavelength of $850 \mathrm{~nm}$. In both cases various linear fill factors are studied in order to maximize the frequency range of a superprism effect. In a double glass structure, the maximum normalized frequency range is equal to 0.012 , while for the air glass structures it reaches 0.108 .
\end{abstract}

Photonic crystals (PCs) are wavelength scaled periodic structures composed of two types of a dielectric [1]. Their properties are defined by the lattice type (hexagonal, rectangular), lattice constant, filling factor and the contrast of a refractive index. By varying these parameters one can change the frequencies that are allowed to propagate inside a crystal and the direction of this propagation. The first articles about PCs were published in the late 1980s. [2, 3] and focused on 1D PCs.

PCs are usually developed using lithographic, holographic or self-assembly methods [1]. In this work we consider an alternative stack and draw method widely applied to optical fiber development. The desired photonic structure is assembled from individual glass rods and tubes and by using rods made from different glasses, or mixing rods and tubes, a wide range of structures can be constructed. The prepared preform is then lowered into the furnace inside a drawing tower (Fig. 1). Different diameters of the PC's rods can be achieved by varying temperature and speed with which the preform is pulled out of the furnace. This method allows fabrication of large volume crystals up to $1 \mathrm{~mm}$ in diameter. In this work we investigate the superprism effect in a volumetric two dimensional crystal with a small refractive index contrast.

The dispersion relation between the frequency $(\omega)$ and the wave vector $(\mathrm{k})$ defines the photonic band structure of the PC. Its geometrical representation is an iso-frequency surface for each photonic band. For isotropic materials

*E-mail: rbuczyns@igf.fuw.edu.pl those surfaces are spherical, but in a periodic medium their shape is complex.

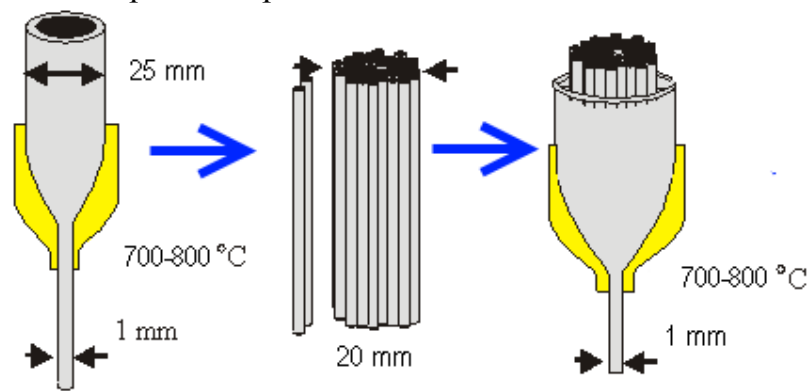

Fig. 1. Schematics of stack and draw fiber production method

This is caused by the fact that iso-frequency surfaces must cross the borders of primitive cells in a reciprocal space at right angles [4]. Furthermore, for photonic crystals the wave vector and the vector of group velocity point in the same direction.

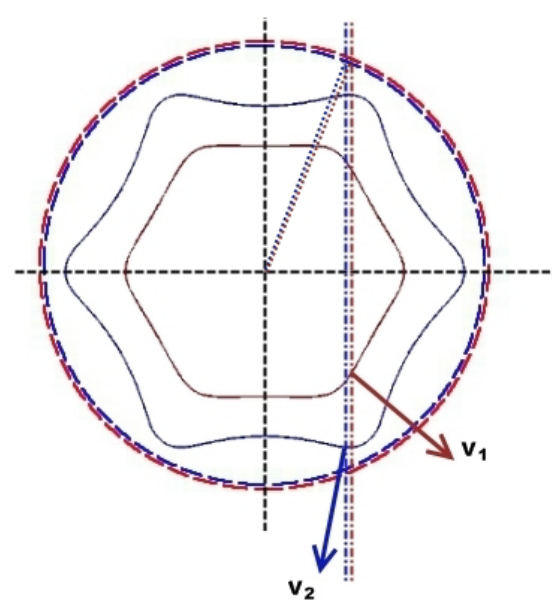

Fig. 2. Dashed circles show iso-frequency surfaces in the air. The solid blue and red lines show iso-frequency lines in a crystal. The dash-dot line represents the conservation of a parallel component of the incident wave vector while the blue and red vectors show different propagation directions for different frequencies.

Since group velocity is a gradient of the dispersion relation, the wave propagation direction inside a photonic crystal is perpendicular to the iso-frequency surface. This, coupled with the translational symmetry which forces 
conservation of the parallel component of the wave vector at the crystal's border, allows the calculation of the direction of propagation for an electromagnetic wave inside a photonic crystal.

A superprism can be used for varying the wave propagation direction, either by changing the angle with which the incident wave enters the crystal or by changing its wavelength. For wavelength demultiplexing, used in telecommunications, the former method is more useful. According to the Sellmeier equation, the medium's refractive index changes with the wavelength. This means that iso-frequency curves are different for different wavelengths, which in turn means a different propagation direction inside the crystal [5].

Usually there is more than one point at which the construction line, defined by the parallel component of the wave vector, crosses the iso-frequency surface. Only those that define propagation vectors away from the crystal border are viable. Furthermore, for non-square lattices or crystal borders which do not follow the crystal main lines, points at which the construction line cross the iso-frequency surface may differ in subsequent Brillouin zones. From the Bloch function it can be deduced that those cross points can be reduced into the first Brillouin zone [5], increasing the number of viable cross points, and in turn increasing the number of directions in which the wave can propagate inside the crystal. Because in a superprism we are interested in one propagation direction, this effect must be avoided.

The MIT Photonic Bands (MPB) package is an iterative eigensolver used to compute the definitefrequency eigenstates of Maxwell's equations in photonic crystals using a plane wave basis. Using the frequency domain method is favorable because we are interested in finding the superprism effect for the lowest possible band. This method allows us to obtain the frequencies and eigenstates at the same time, which helps to quickly differentiate modes and create band diagrams. Because each additional band for the given frequency means additional propagation directions, we focus on frequencies around the photonic band gaps where only one band for the given frequency can be found. After determining the frequency and corresponding mode, MPB is used to calculate the iso-frequency surfaces for given parameters. For the first band there is also a frequency range where no other bands are observed, but the isofrequency surfaces for the first band are too regular and the superprism effect cannot be obtained.

The PC we use as a starting point for this work is manufactured by the Institute of Electronic Materials Technology. It is constructed of the $\mathrm{NC} 21$ and $\mathrm{F} 2$ glasses with refractive indices $\mathrm{n}_{1}=1.5018$ and $\mathrm{n}_{2}=1.6029$, respectively. As a result, a contrast of over 0.1 is obtained. The lattice constant is $0.8 \mu \mathrm{m}$ and the inclusion diameter is equal to $0.4 \mu \mathrm{m}$. Based on the simulations for the in-plane propagation we conclude that the existing photonic band gaps are not in the vicinity of $850 \mathrm{~nm}$.

Similar simulations were performed for a range of PC parameters for similar glasses and lattice types. First we simulated air-glass structures which have the highest refractive index contrast of 0.6029 , and therefore the highest probability of finding photonic band gaps. As expected, the highest frequency range for the superprism effect can be seen for this structure (Fig 3).

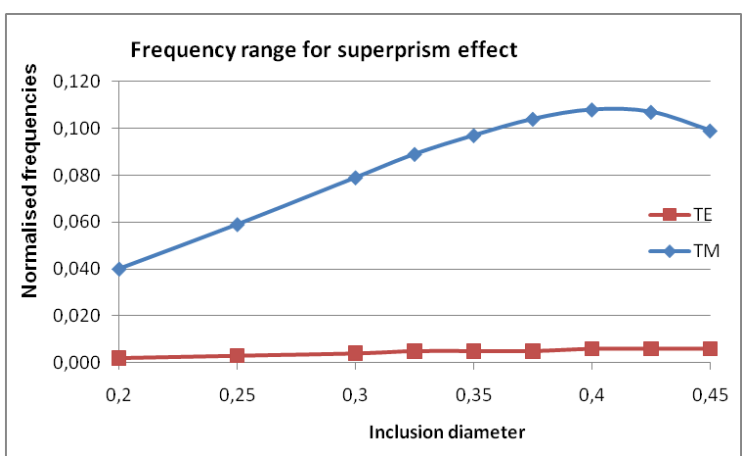

Fig.3. Frequency range for the superprism effect in a glass-air structure depending on a linear fill factor.

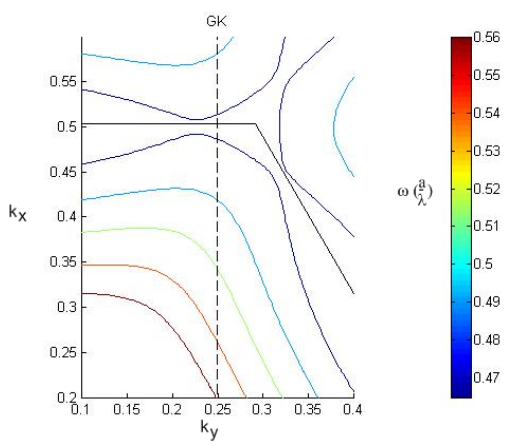

Fig. 4. Iso-frequency curves for the linear fill factor 0.35 and the polarization TM. Curves show a frequency range from 0.464 to 0.561 .

For TM polarization, the frequency range reached 0.1 normalized frequency $(\mathrm{a} / \lambda)$ for the linear fill factor between 0.35 and 0.45 , with a maximum of 0.108 for the fill factor 0.40 (Fig. 4, 5). For TE polarization the maximum frequency range $(0.006)$ is observed for fill factors between 0.4 and 0.45 (Fig. 6, 7).

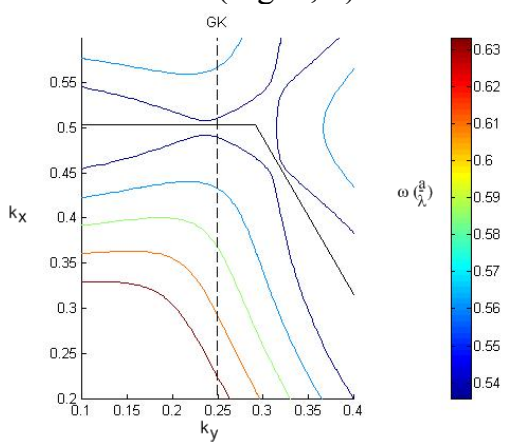


Fig. 5. Iso-frequency curves for the linear fill factor 0.45 and the polarization TM. Curves show frequency range from 0.535 to 0.634 .

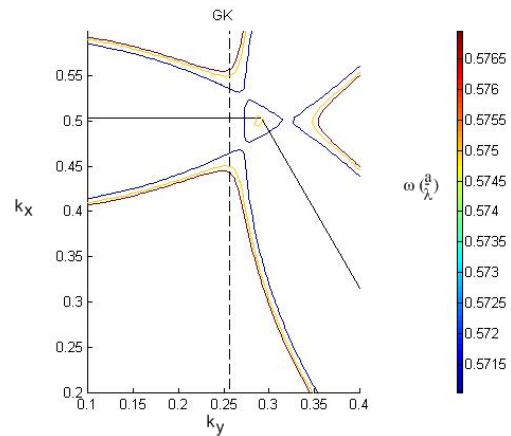

Fig. 6. Iso-frequency curves for the linear fill factor 0.40 and the polarization TE. Curves show frequency range from 0.571 to 0.577 .

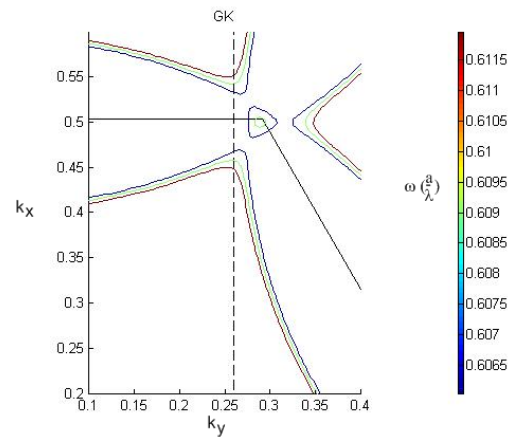

Fig. 7. Iso-frequency curves for the linear fill factor 0.45 and the polarization TE. Curves show frequency range from 0.606 to 0.612 .

Next simulations were performed for theoretical structures based on the PG-3 crystal with different lattice constants. The linear fill factor changes from 0.2 to 0.45 . For the TM polarization, a superprism can be observed for a maximum wavelength span equal to 0.002 normalized frequencies for all linear fill factors (Fig 8). For the TE polarization the observed superprism reaches 0.005 normalized frequencies for a linear fill factor between 0.275 and 0.350 (Fig. 9, 10).

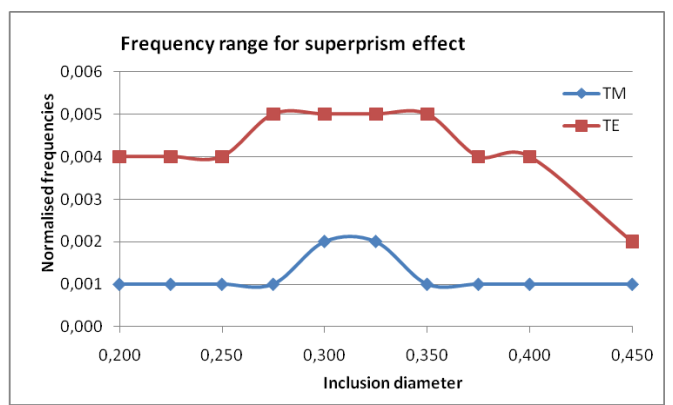

Fig. 8. Frequency range for the superprism effect in the PG-3 based crystal depending on the linear fill factor.

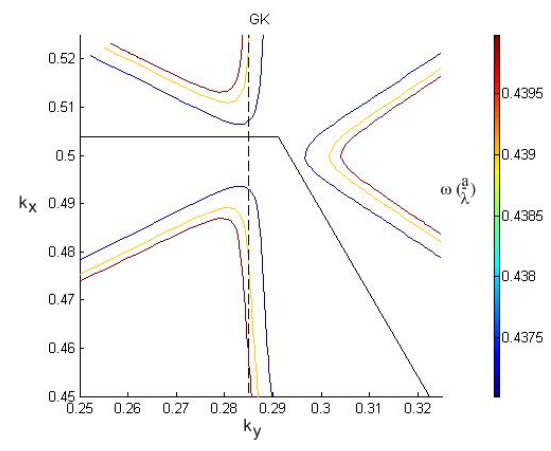

Fig. 9. Iso-frequency curves for the linear fill factor 0.275 and the polarization TE. Curves show frequency range from 0.435 to 0.440 .

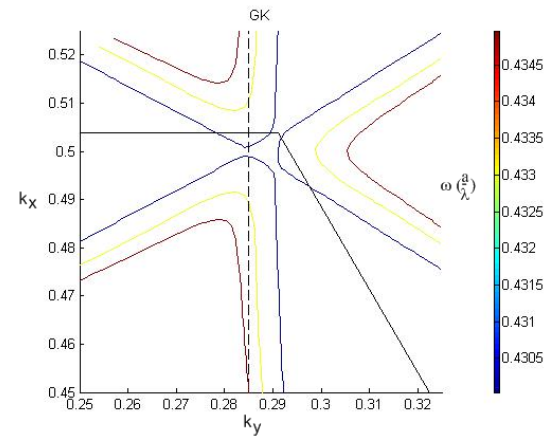

Fig. 10. Iso-frequency curves for the linear fill factor 0.350 and the polarization TE. Curves show frequency range from 0.430 to 0.435 .

In an air glass structure the superprism effect range spans from $814 \mathrm{~nm}$ to $993 \mathrm{~nm}$ for the TM polarization, while for the TE polarization it spans from $845 \mathrm{~nm}$ to 854 $\mathrm{nm}$. For the double glass structure the superprism effect ranges from $850 \mathrm{~nm}$ to $859 \mathrm{~nm}$ for the TE polarization and for the TM polarization from $850 \mathrm{~nm}$ to $853 \mathrm{~nm}$. Based on the obtained results we can conclude that the use of low contrast structures made of two types of glasses allows the development of volumetric photonic crystals with a superprism effect for limited frequency range.

\section{References}

[1] J.-M. Lourtioz, H. Benisty, V. Berger, J.-M. Gerard, D. Maystre, A. Tchelnokov, Photonic Crystals: Towards Nanoscale Photonic Devices, Springer Berlin, 2005.

[2] P. St. J. Russell, Phys. Rev. A 33, 3232-3242 (1986).

[3] R. Zengerle, J. Mod. Opt. 34, 1589 1589-1617 (1987).

[4] P. Yeh, J. Opt. Soc. Am. 69, 742-756 (1979.

[5] N. Malkova, D. A. Scrymgeour, V. Gopalan, Phys. Rev. B 72, 45144 (2005).

[6] S. Foteinopoulou, C. M. Soukoulis, Phys. Rev. B 72, 165112 (2005). 